\title{
Excess Body Weight and Metabolic (Dysfunction)-Associated Fatty Liver Disease (MAFLD)
}

\author{
Elke Roeb \\ Gastroenterology, Justus Liebig University and University Hospital Gießen, Gießen, Germany
}

\author{
Keywords \\ Nonalcoholic fatty liver hepatitis or steatohepatitis (NASH) . \\ Fibrosis · Nonalcoholic fatty liver disease (NAFLD) . \\ Metabolic-associated fatty liver disease (MAFLD) · Fatty \\ liver - Metabolism
}

\begin{abstract}
Background: Nonalcoholic fatty liver disease (NAFLD) describes a continuum of liver abnormalities from simple nonalcoholic fatty liver (NAFL) to nonalcoholic fatty liver hepatitis or steatohepatitis (NASH) to NASH fibrosis. It has a variable course, but just like alcoholic fatty liver disease, it can lead to liver cirrhosis and cancer (hepatocellular carcinoma). Summary: NAFLD is a clinical entity characterized by the presence of liver steatosis, which affects at least $5 \%$ of hepatocytes. Affected are people who consume little or no alcohol and who have no secondary cause of liver steatosis such as viral hepatitis, drug intake (e.g., tamoxifen, amiodarone, methotrexate, etc.), or lipodystrophy. NAFLD is, nowadays, the most common liver disease in Europe, with an estimated prevalence of $25 \%$. The currently widely recognized recommendation for the therapy of NAFLD is a lifestyle modification with the goal of weight loss. Although no drugs are currently approved for the treatment of NAFLD, several candidates are in clinical trials. Besides weight loss and physical activity, corresponding single active ingredients or combination therapies are intended to stop the progression of the disease and, in the best case, reverse it. The newly propagated name MAFLD (metabolic-associated fatty liver disease) should indicate that the disease is associated with metabolic disorders. The term MAFLD also implies multiple over-
\end{abstract}

lapping causes and drivers of this soaring disease. Key Messages: The prevalence of NAFLD continues to rise worldwide. NAFLD, NASH, and fibrosis in NAFLD occur predominantly in patients with obesity and type 2 diabetes (T2DM) or else precede these conditions. The progression of NAFLD is highly dependent on changes in glucose, lipid metabolism, and fibrogenesis. A new definition and nomenclature of fatty liver disease, "metabolic associated fatty liver disease" (MAFLD), should be discussed carefully, since around $40 \%$ of the global population with NAFLD are classified as non-obese and almost $1 / 5$ as lean. Since the pathogenesis of fatty liver disease, obesity, and glucose and lipid metabolism diseases are very closely related, it is important to continue to look for mechanisms that these diseases have in common and develop new therapeutic approaches.

(c) 2021 The Author(s) Published by S. Karger AG, Basel

\section{Introduction}

In 1986, the term nonalcoholic fatty liver disease (NAFLD) was introduced by Schaffner [1]. NAFLD is a clinical entity characterized by the presence of liver steatosis, which affects at least $5 \%$ of hepatocytes. Compromised are people who consume little or no alcohol and who have no secondary cause of liver steatosis such as viral hepatitis (especially HCV), Wilson's disease, drug intake (e.g., tamoxifen, amiodarone, methotrexate, etc.), or lipodystrophy $[2,3]$. NAFLD is, nowadays, the most common liver disease in Europe with an estimated prevalence of 25\% [4]. Fatty liver disease (steatosis hepatis) is present if, histologically, $>5 \%$ of the hepatocytes show an 
accumulation of triglycerides (steatosis), or if the liver fat content on magnetic resonance (MR) spectroscopy or quantitative MR tomography (MRT) is > 5\% [2]. Nonalcoholic steatohepatitis (NASH) is characterized by additional inflammatory changes, and it represents the progressive form of NAFLD with an increased risk of developing fibrosis, cirrhosis, and hepatocellular carcinoma (HCC) [3].

Macrovesicular fat is assessed as a central component of NAFLD grading. If, in addition to fat, there are also (obesity-associated) inflammatory foci and so-called ballooning of the hepatocytes, NASH is present [2]. Ballooning is defined as swelling and rounding of the hepatocytes. It is often associated with small drops of fat. Fibrosis that occurs as a result of steatohepatitis often begins in the center of the lobule in the form of a perivenular and perisinusoidal fibers (fibrosis of the wire mesh type). As the disease progresses, portal fibrosis develops, with the formation of bridging (portoportal and portocentral) septa and, ultimately, cirrhosis of the liver [2].

Depending on the alcohol consumption, a distinction is made between NAFLD and alcoholic fatty liver disease (ALD). Since the threshold dose for alcohol toxicity to the liver varies from person to person, the guidelines do not contain standardized daily alcohol limits for differentiating between NAFLD, ALD, and mixed forms (10-20 g per day for women; 20-30 g per day for men [2]).

A panel of experts recently proposed a simplified algorithm for diagnosing the metabolically associated form of fatty liver disease and renamed it "metabolic dysfunctionassociated fatty liver disease" (MAFLD) [5]. MAFLD can be diagnosed if, in the presence of steatosis (diagnosed using histology, imaging, or biomarkers), 1 of 3 additional criteria is also met:

1. Overweight/obesity

2. Type 2 diabetes mellitus (T2DM)

3. Evidence of metabolic dysregulation, which includes parameters such as waist circumference, blood pressure, serum triglyceride levels, and insulin resistance [5].

Excessive alcohol consumption and the presence of other liver diseases that favor the development of steatosis do not rule out a diagnosis of MAFLD [5]. Patients with T2DM and/or obesity have a significantly increased NAFLD prevalence rate of $60-90 \%$ [6]. While NAFLD/ MAFLD doubles diabetes-associated mortality rates, the presence of T2DM increases the likelihood of NAFLD/ MAFLD progression [6, 7]. The name MAFLD takes these observations into account as well as the close association between metabolic dysfunction, metabolic syndrome, and hepatic steatosis.

\section{Epidemiology}

NAFLD is the leading cause of liver disease in Western countries. An increasing prevalence is currently also being observed in developing countries [8]. According to a recent meta-analysis, the prevalence of NAFLD in the US adult population is $31.9 \%$; worldwide, it is about $28 \%$ [4, $6,9]$, with the most affected regions being South America (31\%) and the Middle East (32\%). In addition, some Asian countries have an increased prevalence rate, particularly Japan, where the prevalence exceeds $50 \%$, followed by Korea (almost 50\%), Singapore, India, and China [6]. Similar data are available in the USA and Europe (> 30 and 24\%, respectively) [6,7]. On the other hand, Africa has the lowest prevalence (14\%). Interestingly, the incidence of NAFLD rises steadily with the prevalence of obesity and diabetes, although it is not clear whether fatty liver is the cause or the consequence of a disturbed metabolism [10]. Data from the National Health and Nutrition Examination Surveys show that the prevalence of NASH cirrhosis and NAFLD-associated advanced fibrosis increased 2.5-fold and 2-fold, respectively, in the period 2009-2012 compared to 1999-2002. In addition, NAFLD made up $75.1 \%$ of chronic liver diseases in 1994 2004 [11]. Very recently, a Swedish cohort study with detailed histological data showed that even mild forms of NAFLD are associated with increased mortality [12].

\section{Fatty Liver in Children}

As part of the global obesity pandemic, the incidence and prevalence of NAFLD is also increasing in children. The obesity rate has more than tripled since the 1960 s and is now $18.5 \%$ in the USA or affects 13.7 million children and adolescents [13]. In Germany, the latest data from the 2nd wave of the KIGGS study found a prevalence of $15.4 \%$ for the presence of overweight and $5.9 \%$ for the presence of obesity $[13,14]$.

The prevalence of NAFLD in children and adolescents varies significantly according to the screening method used (transaminases, ultrasound, or biopsy) and the patient population (regional-ethnic, genetic, and environmental differences, gender, and other risk factors). Ultimately, noninvasive biomarkers are missing for the precise recording of NAFLD prevalence. Estimates assume 3-10\% of all children and adolescents in Western industrialized nations [13]. A pooled analysis of $>16,000$ obese children showed a prevalence of $34.2 \%$ ( $95 \%$ CI $27.8-41.2 \%$ ) versus $7.6 \%$ (95\% CI 5.5-10.3\%) in the general pediatric population [15]. In an autopsy study in San Diego, CA, USA, histologically confirmed NAFLD was found in $9.6 \%$ of all the children and adolescents examined; the prevalence of obese nutritional status accounts for 38\% [16]. 


\section{NAFLD in Overweight, Obesity, and Diabetes}

NAFLD is very common in overweight and obese people [17-20]. However, since increased fat mass does not always go hand in hand with a change in metabolism, the prevalence of NAFLD for a given fat mass could also be very variable. In fact, the Tübingen family study on the pathogenesis of diabetes in overweight and obesity found a prevalence of NAFLD of 21 and $42 \%$, respectively, if no prediabetes was present, but 46 and $70 \%$ if prediabetes was diagnosed [21]. Similar frequencies arise when, not only hyperglycemia, but a global metabolic disorder consisting of hyperglycemia, dyslipidemia, and arterial hypertension was used to stratify people with overweight and obesity. Correspondingly, the prevalence of NAFLD is also increased with normal weight if there is any form of prediabetes (11 vs. $3 \%$ ) or a global metabolic disorder (19 vs. 3\%) [21]. Among people with diabetes, NAFLD was found in $55.5 \%$ and NASH in $37.3 \%$; when stratified according to their BMI, a higher NAFLD prevalence was found in the presence of overweight and obesity [6].

\section{Pathogenesis of NAFLD}

By investigating the most important factors that cause NAFLD, it is helpful to visualize the pathogenesis of glucose and lipid metabolic diseases, which primarily evolve from an unhealthy lifestyle. In addition to a positive energy balance, there is also an increased intake of glucose, fructose, and saturated fatty acids. This leads to an increase in hepatic lipogenesis and subclinical inflammation, particularly in the adipose tissue, intestines, and liver. This metabolic inflammation in adipose tissue and the intestine mediates an increase in hepatic lipogenesis and inflammation through dysregulation of cytokines, fatty acids, the intestinal microbiome and a deterioration of the intestinal barrier [19]. Furthermore, presumably due to the increased hepatic lipid content, there is an increased hepatic mitochondrial respiration in the beginning, which indicates mitochondrial adaptation to this situation. In the further course, and especially in the case of NASH, mitochondrial respiration drops again, and mitochondrial dysfunction as well as oxidative stress occur [22]. These processes determine increased hepatic inflammation, but also hepatic fibrogenesis. Thereby saturated fatty acids, ceramides and diacylglycerol are able to provoke hepatic fat metabolism disorders by hepatic insulin resistance but also intensified hepatic gluconeogenesis.

Hyperglycemia, which is partly caused by insulin resistance of the skeletal muscles and/or an insulin secretion disorder, intensifies the abovementioned processes. In the early phase of type 2 diabetes, hyperglycemia also leads to an increased secretion of insulin, which, in turn, induces lipogenesis in the liver $[17,19]$. Dysregulation of the production and secretion of hepatokines (e.g., fetuin A) increases the inflammation of the adipose tissue and induces insulin resistance [23]. If this metabolic imbalance meets a genetic susceptibility for a disproportionate accumulation of adipose tissue, the development of NAFLD and its progression are accelerated. During aging and the associated change in sex hormone production, there is a redistribution of adipose tissue from the gluteofemoral to the abdominal area. Hypertrophy of adipocytes is most likely mediated by their impaired differentiation in the subcutaneous adipose tissue. The associated increased lipolysis in adipose tissue leads to an enhanced accumulation of lipids in the liver. This process is exacerbated by an unhealthy and high-calorie diet as well as reduced physical activity [2]. Also, an acquired lipodystrophy, mediated by inflammation of the subcutaneous adipose tissue, or caused by therapy with immune checkpoint inhibitors, can be involved in these pathophysiological processes [24]. Genetically determined increased hepatic lipogenesis and fibrogenesis depict additional risk factors in the pathogenesis of NAFLD [25]. On the other hand, increased glucose production, a dysregulation of the synthesis and secretion of cytokines, lipids, and hepatokines, and any prothrombotic status can lead to a worsening of the glucose metabolism and the development of cardiovascular diseases.

In addition, there is a close interaction between the liver and the intestine, in which eubiosis or dysbiosis of the enteral microbiome play an important role. The dysbiosis of the enteral microbiome leads to alteration of the intestinal barrier and the transfer of cellular and molecular (danger- and pathogen-associated molecular patterns [DAMPs and PAMPs]) messenger substances to the liver. In the liver, this is followed by the activation of macrophages and hepatic stellate cells as well as an affection of the hepatic metabolism, with consecutive progression of fatty liver disease, which can lead to NASH, liver fibrosis, liver cirrhosis, and finally HCC. Figure 1 illustrates the main pathophysiological pathways of NAFLD and presents typical histological features. A targeted influencing of the microbiome represents a promising approach for the treatment of metabolic diseases such as NAFLD/ NASH. The possibilities and limitations of probiotic interventions, antibiosis, and fecal microbiome transfer (FMT) have been examined in clinical studies, but not yet included in guidelines $[2,3]$. Important NAFLD risk factors are summarized in Table 1.

\section{Metabolic-Associated Fatty Liver Disease}

Since NAFLD, especially NASH and fibrosis, is very often diagnosed in the context of overweight, obesity, and glucose and lipid metabolism disorders, and the patho- 


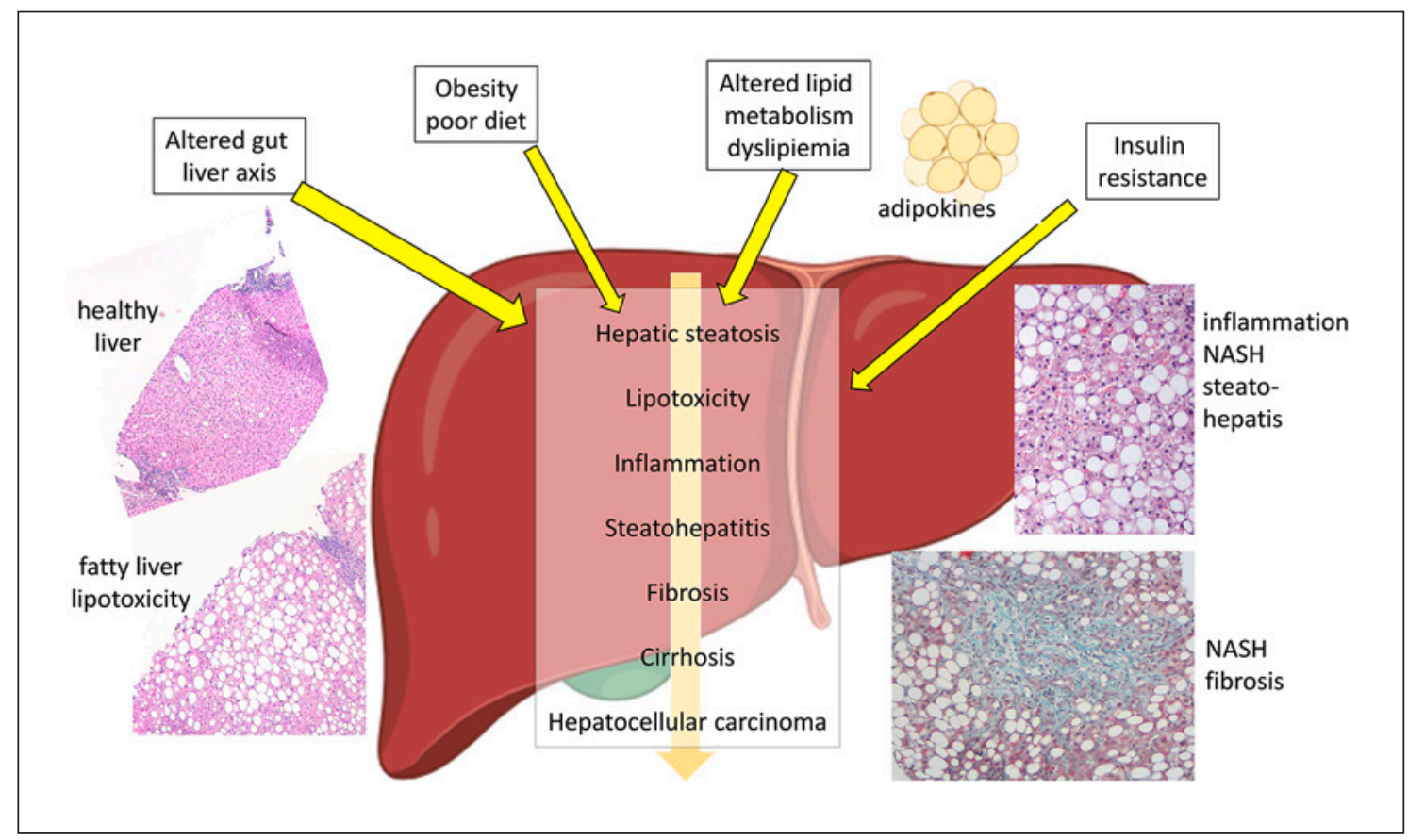

Fig. 1. Pathophysiological aspects of NAFLD and histological examples of NAFL, NASH, and NASH fibrosis. $\mathrm{H} \& \mathrm{E}$ staining of liver sections are presented on the left side. Inflammatory cells (blue) assembled around injured hepatocytes (upper right panel). Moderately enhanced chicken-wire fibrosis was stained (lower right panel).

Table 1. Risk factors for NAFLD (according to Friedman et al. [19] and Lin et al. [26])

\begin{tabular}{lll}
\hline Dietary contributors & $\begin{array}{l}\text { Hepatocellular mechanisms } \\
\text { including adipokines }\end{array}$ & Others/genetic risks \\
\hline Fats & mitochondrial $\beta$-oxidation & uric acid \\
\hline Sugars (fructose) & peroxisomal $\beta$-oxidation & hypoxia (e.g., sleep apnea) \\
\hline Insulin resistance diabetes & lipotoxicity & sedentary lifestyle \\
\hline Obesity & ER stress & hepatic stellate cell activation \\
\hline Metabolic syndrome & oxidative stress & macrophage activation \\
\hline Dysbiosis of intestinal flora & inflammasome activation & genetic polymorphisms (PNPLA3, HSD17B13, \\
\hline Mucosal inflammation, loss of tight junctions & apoptosis & TM6SF2, MBOAT7, GCKR...) \\
\hline $\begin{array}{l}\text { Increased intestinal permeability to gut } \\
\text { microbiota, bacterial translocation }\end{array}$ & necroptosis & gender \\
\hline Endotoxins absorbed to the liver & release of cytokines and chemokines & age \\
\hline Activation of toll-like receptors & $\begin{array}{l}\text { IL-6 (interleukin 6) } \\
\text { TNF- } \alpha \text { (tumor necrosis factor } \alpha)\end{array}$ & \\
\hline & $\begin{array}{l}\text { CCL2 (chemokine C-C motif ligand 2) } \\
\text { PAI-1 (plasminogen activator inhibitor 1) }\end{array}$ & \\
\hline & $\begin{array}{l}\text { leptin } \\
\text { angiotensinogen }\end{array}$ & \\
\hline
\end{tabular}

Summarized here are environmental factors, genetic components, and changes in the intestinal microbiome which influence the development of hepatic steatosis and the progression of NAFLD. NAFLD is characterized as $>5 \%$ triglyceride accumulation in hepatocytes. This can be isolated hepatic steatosis or accompanied by minimal inflammation within the lobules. An altered gut-liver-axis, enhanced susceptibility for hepatic triglyceride accumulation, altered lipid metabolism, dyslipidemia, and insulin resistance are the key components of NAFLD pathophysiology. 
genesis of these diseases is closely interconnected, many experts in the field of NAFLD research are now calling for a new definition and a change in terminology [5]. Advanced liver fibrosis and the NAFLD fibrosis score are independent risk factors for cardiovascular diseases, even after considering traditional risk factors and cardiovascular risk scores. In a prospective study, cardiovascular events could be predicted quite accurately [27].

However, it is justifiably stated that fatty liver often occurs in patients with liver diseases, which previously served to rule out NAFLD. The course of these diseases (e.g., tamoxifen-induced fatty liver and acute pregnancy fatty liver) is also significantly influenced by the presence of fatty liver. It is also stated that many patients develop liver fibrosis and/or HCC without going through the NASH stage, i.e., inflammation. This means that hepatic inflammation would cease to exist as an independent entity within NAFLD.

In patients with NAFLD, any additional component of the metabolic syndrome increases the risk of cirrhosis and HCC. Diabetes is associated with the highest risk of progression to HCC. Patients with T2DM and simultaneous arterial hypertension and obesity could therefore be an important target cohort for secondary prevention [28].

Finally, it should be noted that it is very difficult to control for alcohol consumption (especially a long time ago) to be able to quantify precisely, and that a brisk demand for this usually has a negative impact on the doctorpatient conversation.

If this new definition and nomenclature "metabolic (dysfunction)-associated fatty liver disease" (MAFLD) prevails and is recognized by medical societies, then overweight, obesity, diabetes, and/or metabolic disorders (parameters of the metabolic syndrome, insulin resistance, and increased concentrations of C-reactive protein), in addition to steatosis as a diagnostic parameter of fatty liver disease, will come to the fore.

In a recent systematic review and meta-analysis, however, around $40 \%$ of the global population with NAFLD was classified as nonobese and almost $1 / 5$ as lean. Both nonobese and lean individuals had significant long-term liver-associated and nonliver comorbidities. These results suggest that obesity should not be the only criterion for NAFLD screening. In addition, clinical studies on NAFLD treatment should include subjects from all BMI categories [29]. Taken together, the chapter on correct designation of NAFLD is not yet closed.

\section{Genetic Factors and MAFLD}

The results of several studies suggest that around 50\% of observed interindividual differences in liver fat content are influenced by genetic factors [30]. In recent years, var- ious genetic risk variants have been identified that influence the development and/or course of steatosis, including cirrhosis and the development of HCC [30]. These genetic risk factors include variants in the genes: PNPLA3 (patatin-like phospholipase-containing 3), TM6SF2 (transmembrane 6 superfamily member 2), MBOAT7 (membrane-bound O-acyltransferase domain-containing 7), GCKR (glucokinase regulator), and HSD17B13 (hydroxysteroid $17 \beta$ dehydrogenase 13 ).

With increasing BMI, there is an increased risk of developing fatty liver mediated by the PNPLA3, TM6SF2, and GCKR gene variants [31]. For carriers of the PNPLA3 risk variant ( $M$ variant), with increasing body weight, there is also an increase in the ALT values and the risk of progression to cirrhosis [31]. Conversely, the influence of the protective $H S D 17 B 13$ variant is particularly pronounced in obese individuals [32]. Patients with NAFLD/ MAFLD are more likely to be insulin-resistant than people without fatty liver, even if they are not overweight and do not have any diabetic metabolic condition. Insulin resistance, in turn, increases the risk of developing NAFLD/ MAFLD [2]. High insulin levels also increase the influence of the PNPLA3 risk allele on the development of steatosis [33]. Thus, both obesity and increased insulin levels potentiate the influence of the PNPLA3 risk variant on NAFLD/MAFLD development and its progression. The cardiovascular risk is reduced in carriers of the PNPLA3 and TM6SF2 variants by reducing the serum triglyceride and lipid levels, but it is increased in carriers of the GCKR risk variant through increased serum lipid levels [34]. Genetic variants thus influence the development of different phenotypes of the metabolic syndrome (obesity, hyperlipidemia, T2DM, cardiovascular risk, and MAFLD).

The prevalence of NAFLD in pregnancy has nearly tripled in the past decade and is independently associated with hypertensive complications, hemorrhage postpartum, and premature delivery. NAFLD should therefore be viewed as a high-hazard obstetric disease and affect counseling and prenatal care [35].

\section{Treatment Strategies}

\section{Lifestyle Intervention}

The currently widely recognized recommendation for the therapy of NAFLD is a lifestyle modification with the goal of weight loss. A weight reduction of even $5 \%$ of body weight can lead to a reduction of liver fat content, and a weight loss of $\geq 10 \%$ can significantly improve existing liver fibrosis $[2,3,36]$. In a large, prospective cohort study to simultaneously evaluate the impact of obesity and physical activity on the long-term risk of liver-related mortality, Simon et al. [37] demonstrated that a high level of physical activity lowers obesity-related mortality. 
However, it is difficult for most patients to achieve this weight loss and then maintain it for life [17]. A diet rich in protein but low in calories could significantly reduce liver fat. According to this relatively small study, proteins have an inhibitory effect on fat absorption and lipid synthesis [38]. Such findings could influence nutritional recommendations in the future.

\section{Endoscopy and Bariatric Surgery}

In patients unresponsive to lifestyle interventions, bariatric surgery is an option for long-term weight loss. The oftentimes severe weight loss leads to a reduction in liver fat and improves all histological components of NAFLD, including the degree of fibrosis $[3,36]$. However, there are also obvious limitations, including possible complications from the operation, poor patient compliance, the availability of bariatric centers, and the resulting costs for the health system [36]. Contraindications for metabolic surgery are uncontrolled cancer or autoimmune diseases, a risk of surgery and anesthesia that is too high (e.g., high-grade cardiopulmonary insufficiency), uncontrolled psychoses and severe eating disorders (especially untreated bulimia), inadequate psychosocial stability (e.g., in the case of insurmountable difficulties, lack of a permanent residence, and nonaffordable life-long supplementation), an existing (not a planned) pregnan$\mathrm{cy}$, and insufficient motivation and compliance of the patient, especially with regard to a necessary lifestyle change. A very advanced stage of liver cirrhosis (especially CHILD stage $\mathrm{C}$ ) is also considered a contraindication (not CHILD stages A and B).

Gastrointestinal endoscopy is playing a growing role before, after, or as an alternative to metabolic surgery. Postoperative problems such as insufficiency of gut sutures or dumping syndrome can be treated endoscopically. Biliary access after bariatric surgery requires precise knowledge of the changed anatomy. In addition to the gastric balloon, the endoluminal tube bypass or the duodenal mucosal resurfacing, other endoscopic procedures are being developed that will be considered in the future as bridging or, in mild cases, as an alternative to surgery [39].

\section{Pharmacological Therapies}

The prevention of obesity and physical inactivity targets the most important risk factors. Physical activity has been shown to improve fatty liver even without reducing body weight. For patients with T2DM and hyperlipidemia, established pharmacological treatment has shown significant effects on NASH. However, up to now there is no specific drug therapy approved for NASH or NAFLD.

A number of new substances are currently in phase II and III of clinical development [40]. Four of these compounds are currently in ongoing phase III trials, the nu- clear bile acid receptor FXR agonist obeticholic acid (already approved for primary biliary cholangitis $[\mathrm{PBC}]$ ), cenicriviroc, a CCR2/CCR5 chemokine antagonist, aramchol, an SCD-1 inhibitor, and resmetirom, a thyroid hormone receptor $\beta$ agonist. A concise overview is presented in Roeb and Geier [40] and 45 studies were found in the NIH US National Library of Medicine publication [41].

The results of a planned interim analysis of a phase III study with obeticholic acid in NASH showed a histological improvement in fibrosis as well as in the key components of NASH. Long-term proof of effectiveness is still pending [42].

As treatment can only be offered to those patients with identified disease, diagnostic algorithms need to be applied on a broader basis in primary care. A prerequisite will be the availability of respective diagnostic platforms for advanced NAFLD testing.

\section{Conclusion}

Fatty liver disease is a major problem for the development of advanced liver diseases, such as liver cirrhosis and HCC. Furthermore, if fatty liver disease is present, there is a high risk of developing T2DM and cardiovascular diseases. Since the pathogenesis of fatty liver disease, obesity, and the metabolic diseases mentioned is very closely related, it is important to continue to search for mechanisms that these diseases have in common and develop new therapeutic approaches, as their close interlinking will probably also lead to a new definition of fatty liver diseases.

\section{Conclusion for General Practitioners}

- The prevalence of NAFLD continues to rise worldwide.

- NAFLD, NASH, and fibrosis in NAFLD occur predominantly in obesity and T2DM or precede them.

- The progression of NAFLD is highly dependent on changes in glucose metabolism, lipid metabolism, and fibrogenesis.

- A new definition and nomenclature of a form of fatty liver disease, "metabolic (dysfunction)-associated fatty liver disease" (MAFLD), should be discussed carefully, since around $40 \%$ of the global population with NAFLD are classified as nonobese and almost $1 / 5$ as lean. However, $40 \%$ of nonobese NAFLD patients in the meta-analysis published by Ye et al. [29] were just as metabolically unhealthy as obese people with NAFLD, and the definition MAFLD indeed includes lean patients with metabolic risk factors [5]. Up to 
now, all FDA/EMA-accepted end points rely on the published NAFLD definition, so it seems practically impossible to implement a new name without challenging the search for new therapeutics.

- Since the pathogenesis of fatty liver disease, obesity, and glucose and lipid metabolism diseases are very closely related, it is important to continue to look for common mechanisms for the development of and therapeutic approaches for these diseases.

\section{Conflict of Interest Statement}

There were no conflicts of interest.

\section{Funding Sources}

The work was funded by grants from the German Research Foundation (RO 957/11-1), the University Hospital Giessen and Marburg ( $\$ 2$ Abs 3 No. 10/2013 GI), and the von Behring Röntgen Foundation (66-0008).

\section{References}

1 Schaffner F, Thaler H. Nonalcoholic fatty liver disease. Prog Liver Dis. 1986;8:283-98.

2 Roeb E, Steffen HM, Bantel H, Baumann U, Canbay A, Demir M, et al. [S2k Guideline non-alcoholic fatty liver disease]. Z Gastroenterol. 2015 Jul;53(7):668-723.

3 European Association for the Study of the Liver (EASL)European Association for the Study of Diabetes (EASD)European Association for the Study of Obesity (EASO). EASLEASD-EASO Clinical Practice Guidelines for the management of non-alcoholic fatty liver disease. J Hepatol. 2016 Jun;64(6):1388-402.

4 Younossi ZM. Non-alcoholic fatty liver disease - A global public health perspective. J Hepatol. 2019 Mar;70(3):531-44.

5 Eslam M, Newsome PN, Sarin SK, Anstee QM, Targher G, Romero-Gomez M, et al. A new definition for metabolic dysfunction-associated fatty liver disease: an international expert consensus statement. J Hepatol. 2020 Jul;73(1):202-9.

6 Younossi ZM, Golabi P, de Avila L, Paik JM, Srishord M, Fukui N, et al. The global epidemiology of NAFLD and NASH in patients with type 2 diabetes: A systematic review and meta-analysis. J Hepatol. 2019 Oct;71(4): 793-801.

7 Alvarez CS, Graubard BI, Thistle JE, Petrick JL, McGlynn KA. Attributable Fractions of Nonalcoholic Fatty Liver Disease for Mortality in the United States: Results From the Third National Health and Nutrition Examination Survey With 27 Years of Follow-up. Hepatology. 2020 Aug;72(2):430-40.

8 Vernon G, Baranova A, Younossi ZM. Systematic review: the epidemiology and natural history of non-alcoholic fatty liver disease and non-alcoholic steatohepatitis in adults. Aliment Pharmacol Ther. 2011 Aug;34(3):27485.

9 Younossi ZM, Stepanova M, Younossi Y, Golabi P, Mishra A, Rafiq N, et al. Epidemiology of chronic liver diseases in the USA in the past three decades. Gut. 2020 Mar;69(3):564-8.

10 Zawdie B, Tadesse S, Wolide AD, Nigatu TA, Bobasa EM. Non-Alcoholic Fatty Liver Disease and Associated Factors among Type 2 Diabetic Patients in Southwest Ethiopia Ethiop J Health Sci. 2018 Jan;28(1):19-30.
11 Kabbany MN, Conjeevaram Selvakumar PK, Watt K, Lopez R, Akras Z, Zein N, et al. Prevalence of Nonalcoholic Steatohepatitis-Associated Cirrhosis in the United States: An Analysis of National Health and Nutrition Examination Survey Data. Am J Gastroenterol. 2017 Apr;112(4):581-7.

12 Simon TG, Roelstraete B, Khalili H, Hagström H, Ludvigsson JF. Mortality in biopsyconfirmed nonalcoholic fatty liver disease: results from a nationwide cohort. Gut. 2020, Online ahead of print.

13 Nobili V, Alisi A, Valenti L, Miele L, Feldstein AE, Alkhouri N. NAFLD in children: new genes, new diagnostic modalities and new drugs. Nat Rev Gastroenterol Hepatol. 2019 Sep;16(9):517-30.

14 Mann JP, Valenti L, Scorletti E, Byrne CD, Nobili V. Nonalcoholic Fatty Liver Disease in Children. Semin Liver Dis. 2018 Feb;38(1): $1-13$.

15 Bellentani S, Dalle Grave R, Suppini A, Marchesini G; Fatty Liver Italian Network. Behavior therapy for nonalcoholic fatty liver disease: the need for a multidisciplinary approach. Hepatology. 2008 Feb;47(2):746-54.

16 Schwimmer JB, Zepeda A, Newton KP, Xanthakos SA, Behling C, Hallinan EK, et al.; Nonalcoholic Steatohepatitis Clinical Research Network. Longitudinal assessment of high blood pressure in children with nonalcoholic fatty liver disease. PLoS One. 2014 Nov; 9(11):e112569.

17 Tilg H, Moschen AR, Roden M. NAFLD and diabetes mellitus. Nat Rev Gastroenterol Hepatol. 2017 Jan;14(1):32-42.

18 Younossi Z, Anstee QM, Marietti M, Hardy T, Henry L, Eslam M, et al. Global burden of NAFLD and NASH: trends, predictions, risk factors and prevention. Nat Rev Gastroenterol Hepatol. 2018 Jan;15(1):11-20.

19 Friedman SL, Neuschwander-Tetri BA, Rinella M, Sanyal AJ. Mechanisms of NAFLD development and therapeutic strategies. Nat Med. 2018 Jul;24(7):908-22.

20 Stefan N, Häring HU, Cusi K. Non-alcoholic fatty liver disease: causes, diagnosis, cardiometabolic consequences, and treatment strategies. Lancet Diabetes Endocrinol. 2019 Apr; $7(4): 313-24$.

21 Stefan N, Fritsche A, Schick F, Häring HU. Phenotypes of prediabetes and stratification of cardiometabolic risk. Lancet Diabetes Endocrinol. 2016 Sep;4(9):789-98.
22 Pafili K, Roden M. Nonalcoholic fatty liver disease (NAFLD) from pathogenesis to treatment concepts in humans. Mol Metab. 2020, Online ahead of print.

23 Pal D, Dasgupta S, Kundu R, Maitra S, Das G, Mukhopadhyay S, et al. Fetuin-A acts as an endogenous ligand of TLR4 to promote lipidinduced insulin resistance. Nat Med. 2012 Aug;18(8):1279-85.

24 Eigentler T, Lomberg D, Machann J, Stefan N. Lipodystrophic Nonalcoholic Fatty Liver Disease Induced by Immune Checkpoint Blockade. Ann Intern Med. 2020 Jun;172(12):8367.

25 Eslam M, Valenti L, Romeo S. Genetics and epigenetics of NAFLD and NASH: clinical impact. J Hepatol. 2018 Feb;68(2):268-79.

26 Lin YC, Wu CC, Ni YH. New Perspectives on Genetic Prediction for Pediatric Metabolic Associated Fatty Liver Disease. Front Pediatr. 2020 Dec;8:603654.

27 Henson JB, Simon TG, Kaplan A, Osganian S, Masia R, Corey KE. Advanced fibrosis is associated with incident cardiovascular disease in patients with non-alcoholic fatty liver disease. Aliment Pharmacol Ther. 2020 Apr; 51(7):728-36.

28 Kanwal F, Kramer JR, Li L, Dai J, Natarajan Y, Yu X, et al. Effect of Metabolic Traits on the Risk of Cirrhosis and Hepatocellular Cancer in Nonalcoholic Fatty Liver Disease. Hepatology. 2020 Mar;71(3):808-19.

29 Ye Q, Zou B, Yeo YH, Li J, Huang DQ, Wu Y, et al. Global prevalence, incidence, and outcomes of non-obese or lean non-alcoholic fatty liver disease: a systematic review and metaanalysis. Lancet Gastroenterol Hepatol. 2020 Aug;5(8):739-52.

30 Anstee QM, Seth D, Day CP. Genetic Factors That Affect Risk of Alcoholic and Nonalcoholic Fatty Liver Disease. Gastroenterology. 2016 Jun;150(8):1728-44.e7.

31 Stender S, Kozlitina J, Nordestgaard BG, Tybjærg-Hansen A, Hobbs HH, Cohen JC. Adiposity amplifies the genetic risk of fatty liver disease conferred by multiple loci. Nat Genet. 2017 Jun;49(6):842-7.

32 Gellert-Kristensen H, Nordestgaard BG, Tybjaerg-Hansen A, Stender S. High Risk of Fatty Liver Disease Amplifies the Alanine Transaminase-Lowering Effect of a HSD17B13 Variant. Hepatology. 2020 Jan;71(1):56-66. 
33 Barata L, Feitosa MF, Bielak LF, Halligan B, Baldridge AS, Guo X, et al. Insulin Resistance Exacerbates Genetic Predisposition to Nonalcoholic Fatty Liver Disease in Individuals Without Diabetes. Hepatol Commun. 2019 Apr;3(7):894-907.

34 Brouwers MC, Simons N, Stehouwer CD, Isaacs A. Non-alcoholic fatty liver disease and cardiovascular disease: assessing the evidence for causality. Diabetologia. 2020 Feb;63(2): 253-60.

35 Sarkar M, Grab J, Dodge JL, Gunderson EP, Rubin J, Irani RA, et al. Non-alcoholic fatty liver disease in pregnancy is associated with adverse maternal and perinatal outcomes. J Hepatol. 2020 Sep;73(3):516-22.
36 Targher G, Lonardo A, Byrne CD. Nonalcoholic fatty liver disease and chronic vascular complications of diabetes mellitus. Nat Rev Endocrinol. 2018 Feb;14(2):99-114.

37 Simon TG, Kim MN, Luo X, Yang W, Ma Y, Chong DQ, et al. Physical activity compared to adiposity and risk of liver-related mortality: results from two prospective, nationwide cohorts. J Hepatol. 2020 Jun;72(6):1062-9.

38 Xu C, Markova M, Seebeck N, Loft A, Hornemann S, Gantert T, et al. High-protein diet more effectively reduces hepatic fat than lowprotein diet despite lower autophagy and FGF21 levels. Liver Int. 2020 Dec;40(12): 2982-97.
39 Štimac D, Klobučar Majanović S, Belančić A. Endoscopic Treatment of Obesity: From Past to Future. Dig Dis. 2020, Online ahead of print.

40 Roeb E, Geier A. Nonalcoholic steatohepatitis (NASH) - current treatment recommendations and future developments. Z Gastroenterol. 2019 Apr;57(4):508-17.

41 [Internet] NIH US National Library of Medicine. Available from: https://www.clinicaltrials.gov/ct $2 /$ results? cond = NAFLD+ nash\&age_v $=$ \&gndr $=$ \&type $=\&$ rslt $=$ \&phase $=2$ \&Search=Apply

42 Younossi ZM, Ratziu V, Loomba R, Rinella M, Anstee QM, Goodman Z, et al.; REGENERATE Study Investigators. Obeticholic acid for the treatment of non-alcoholic steatohepatitis: interim analysis from a multicentre, randomised, placebo-controlled phase 3 trial. Lancet. 2019 Dec;394(10215):2184-96. 